\title{
Gallium Arsenide Nanowires Formed by Au-assisted Metal-organic Chemical Vapor Deposition: Effect of Growth Temperature
}

\author{
Rosnita Muhammad, Zulkafli Othaman, Yussof Wahab, Samsudi Sakrani, W. Faizal W. Ahmad \& Mohd Nazri \\ Physics Department, Faculty of Science \\ University Teknologi Malaysia \\ 81310 UTM Skudai, Johor, Malaysia \\ E-mail: rosnita@utm.my
}

\begin{abstract}
We have investigated the growth of gallium arsenide (GaAs) nanowires as a function of temperatures in metal organic chemical vapor deposition (MOCVD) to establish the mechanisms that govern wire growth and to optimize growth conditions. The growth follows the vapor-liquid-solid method by applying nanoparticle gold colloid as a catalyst to forms a eutectic liquid alloy with the substrate. The semi insulating undoped (111)B GaAs was first dipped in the poly-L-lysine solution before 30nm gold colloid dropped on the substrate surface. Growth process in the MOCVD system were done at temperatures between 380 and $600^{\circ} \mathrm{C}$ with growth time set is $30 \mathrm{~min}$. All the grown samples were analyzed using a field emmission scanning electron microscope (FE-SEM) and scanning electron microscopy (SEM). With increasing temperature the nanowire height increases but leads to significant tapering of the nanowire due to competing growth at the (111) substrate surface. At low temperatures nanowires grown are cylindrical-shaped with diameter wires between 50 and $100 \mathrm{~nm}$.
\end{abstract}

Keywords: GaAs, Nanowire, Vapor-liquid solid mechanism, MOCVD, Tapering

\section{Introduction}

Nanowires can be grown as highly perfect one-dimensional nano-structures, suitable for basic physics investigation (nano-probes, transport physics, etc) as well as for potential applications in optical and electrical devices (LEDs, waveguides, field emitters, nano-probes, etc)(Lars Samuelson, 2003)(Yue Wu, Yi Chui, Lynn Huynh, Carl J. Barrelet, David C. Bell and Charles M. Lieber, 2004)( L. J. Lauhon, Mark S. Gudiksen and Charles M. Lieber, 2004). In most cases, the growth is initiated by the presence of metal particles, which act as catalyst. In the classical description, the growth follows the vapor-liquid solid (VLS) mechanism(R. S. Wagner and W. C. Ellis, 1964), although details are still in debate. Metal-organic chemical vapor deposition (MOCVD) for growing GaAs and InAs nanowires was already used by Hiruma et al (K. Hiruma, M. Yazawa, T. Katsuyama, K. Ogawa, K. Haraguchi, M. Koguchi and H. Kakibayashi, 1995), with deposited Au films, transforming into catalytically active nanoparticles by annealing. Very recently, the fabrication of nanowire-based one-dimensional heterostructures has been reported (Charles M. Lieber, 2003)(L. J. Lauhon, Mark S. Gudiksen and Charles M. Lieber, 2004) and their functionality in resonant tunneling structures has been demonstrated (M. T. Bjork et. al., 2002). However, there are still many open question to be answered before nanowires can be used as versatile building blocks in nanostructure devices. We focus in this paper on investigating the growth mechanism, with special emphasis on effect of growth temperature and optimizing the growth conditions of GaAs nanowires.

\section{Experiment}

The experiments start with semi insulating undoped GaAs (111)B substrates immersed in $0.1 \%$ poly-L-lysine (PLL) solution for $3 \mathrm{~min}$. After washing with deionize water and subsequent drying with $\mathrm{N}_{2}$, the wafer was cut into desired size. The $30 \mathrm{~nm}$ diameter gold colloids were dispersed on the wafer surface by using microlittre pipette and immediately washed after $20 \mathrm{sec}$. The PLL layer on the surface is positively charged and attracts the negatively charged of the gold colloids. The cutting wafer was put on the graphite susceptor inside the vertical reactor in the MOCVD system for the growth processes. The growth pressure inside vertical reactor chamber was 76 Torr. Trimethylgallium 
(TMGa) and arsine $\left(\mathrm{AsH}_{3} ; 10 \%\right.$ in $\left.\mathrm{H}_{2}\right)$ were used as the source gases. The $\mathrm{AsH}_{3}$ to TMGa molar flow ratio (the V/III ratio) was set at 166. The wafer was annealed in situ at $600^{\circ} \mathrm{C}$ under $\mathrm{AsH}_{3}$ ambient for 10 min to desorbed surface contaminants and form eutectic alloy between $\mathrm{Ga}$ and gold colloid $(\mathrm{Au})$. After annealing process, the temperature was ramped down to the desired nanowire growth temperature. The growth temperature was chosen between $380^{\circ} \mathrm{C}$ to $600^{\circ} \mathrm{C}$. Nanowire growth started when trimethylgallium (TMGa) was supplied to the reactor chamber. The nanowire growth time was kept constant at $30 \mathrm{~min}$. All the grown samples were then characterized using a JSM-6390LV scanning electron microscope (SEM) and JEOL JSM-6701F field emission scanning electron microscope (FE-SEM).

\section{Results and discussions}

Figure 1 shows scanning electron microscopy (SEM) images of GaAs nanowires grown at various temperatures $\left(380^{\circ} \mathrm{C}-\right.$ $600^{\circ} \mathrm{C}$ ), taken with the substrates tilted $30^{\circ}$ from the electron beam. The tilting is needed to verifying structures from $2 \mathrm{D}$ to $3 \mathrm{D}$ images. The first temperature (Figure 1(a)) is closed to the lowest eutectic temperatures $\left(339.4^{\circ} \mathrm{C}\right.$ and $348.9^{\circ} \mathrm{C}$ ) of the AuGa phase diagram (Zhong Lin Wang, Yi Liu and Ze Zhang, 2002) which particles phase transition is solid below $340^{\circ} \mathrm{C}$ and return to liquid phase at $400^{\circ} \mathrm{C}$. At this stage, only particles in size range between $50-100 \mathrm{~nm}$ distributed on the substrate surface. The particles increased further in width and height with increasing growth temperature to $440^{\circ} \mathrm{C}$. The nanowire height increases with increasing temperature up to $540^{\circ} \mathrm{C}$ and then decreases at higher temperature, while tapering of the nanowires increases continuously. From the classical VLS mechanism theory, an ideal liquid surface captures all impinging material source atoms, while a solid surface rejects almost all source atoms if the temperature is sufficiently high(R. S. Wagner and W. C. Ellis, 1964).

Tapering of the nanowires at high growth temperature is due to the effect of radial growth. If the growth of nanowires is dominated by the reaction species impinging directly on the Au nanoparticles on the top of the nanowires, ideal one dimensional nanowire growth is achieved without any tapering. However, a significant amount of reaction species could also have arrived at the nanowire sidewall or (111)B substrate and diffused toward the Au nanoparticle. During the migration process, the incorporation of the reaction species into the (111)B surface and nanowire sidewall takes place at high growth temperature. This incorporation, known as the 2-D growth, results in the tapering (H. H. Tan, K. Sears, S. Mokkapati, Lan Fu, Yong Kim, P. McGowan, M Buda and C. Jagadish, 2006). The decrease of apparent nanowire height at $600^{\circ} \mathrm{C}$ is due to the increase of 2-D growth at (111)B substrate. From Figure 1 the optimized growth temperature is in the range of $420-480^{\circ} \mathrm{C}$ with little tapering and reasonable uniformity.

All the nanowires are growing in $<111>B$ direction i.e., they are standing vertically on the (111)As surface. In addition, also on (110) cleavage planes, which were exposed to Au nanoparticles dropped, nanowires were growing. At lower growth temperature, the nanowires grown are cylindrical shape whereas at high temperature, visualizing the hexagonal cross-section with the $\{110\}$ side facets is clearly defined. Plante and LaPierre (2008) already investigated the faceting GaAs nanowire sidewall using molecular beam epitaxial growth. They conclude that tapered tip exhibit different facets than the base of the wire during planar growth of the nanowire sidewall (M. C. Plante and R. R. LaPierre, 2008).

The temperature-dependent reduction in tapering also can be explained using schematically with reference to the axial and radial growth mechanism shown in Figure 2(Hannah J.Joyce, Qiang Gao, H. Hoe Tan, Chennupati Jagadish, Yong Kim, Xin Zhang, Yanan Guo and Jin Zou, 2007). Reaction species which impinge directly upon the nanoparticle contribute to axial growth. Additionally, Ga adatoms are adsorbed on the substrate and nanowire sidewalls and diffuse along the concentration gradient toward the growing nanoparticle-nanowire interface. These diffusion adatoms contribute to both radial and axial growth, hence radial growth competes with axial growth at high growth temperature as can be seen on Figure 3. Images of GaAs nanowires are taken from cross-sectional FE-SEM measurement. The nanowires get increasingly tapered with the thicker end at the base and segmented. These trends are in agreement with observations of Hiruma et. al. (K. Hiruma, M. Yazawa, T. Katsuyama, K. Ogawa, K. Haraguchi, M. Koguchi and H. Kakibayashi, 1995). Because radial growth is kinetically limited, diffusing adatoms are less likely to be incorporated into nanowire sidewalls at lower growth temperatures. Furthermore, adatom diffusion length decreases with decreasing growth temperature. This reduces the flux of adatoms diffusing from the substrate, limiting radial growth and tapering.

The measured height and width of the GaAs nanowires at different growth temperature were plotted in Figure 4. The height of the nanowires was measured above the base feature, which is an actual measure of the growth enhancement of the catalytic particle. The width and diameter was measured at the middle point of the full length for each wire as did the average length. The nanowire height increases with increasing temperature up to $540^{\circ} \mathrm{C}$ and then decreases at higher temperature. The width of the nanowires increases continuously. Each measurement point represents an average over about 50 nanowires, selected from areas of high nanowires homogeneity. A maximum in nanowire height occurred at $540^{\circ} \mathrm{C}$ growth temperature. Towards low temperatures, the height decreases almost linearly in range $420-540{ }^{\circ} \mathrm{C}$. By plotting the graph of growth rate $(\mathrm{nm} / \mathrm{s})$ versus $1 / \mathrm{T}(\mathrm{K})$ for GaAs nanawire, the slope of the low-temperature times (-R) results in Arrhenius activation energy which is $58.86 \mathrm{~kJ} / \mathrm{mol}$. The equation used was 


$$
\mathrm{E}_{\mathrm{a}}=-\mathrm{R}\left(\frac{\partial \ln k}{\partial\left(\frac{1}{T}\right)}\right)
$$

where $\mathrm{E}_{\mathrm{a}}$ is the activation energy and $\mathrm{R}$ is the gas constant equal to $8.314472 \mathrm{JK}^{-1} \mathrm{~mol}^{-1}$ (James F. Shackelford, 1996). The activation energy, $58.86 \mathrm{~kJ} / \mathrm{mol}$ found agreed with activation energy for the overall GaAs low-temperature MOCVD process (D. H. Reep, S. K. Ghandi, 1983). Borgstrom et al (2004) found in their observation of activation energy at higher growth temperature after limitation of nanowires growth rate, that $\mathrm{Au}$ on top of the nanowires does not effect the activation energy, but the process outside the droplet (M. Borgstrom, K. Depert, L. Samuelson, W. Seifert, 2004).

\section{Conclusion}

We have investigated the height, width and shape of Au-catalyzed GaAs nanowires grown on the (111)B GaAs surface, as a function of temperature using MOCVD system. With increasing temperature, the height of the nanowire increases but lead to tapered of the nanowire sidewall. Towards low temperatures, the nanowire height decreases almost linearly in the arrhenius plotted. The slope results in activation energy about $58.86 \mathrm{~kJ} / \mathrm{mol}$, the value is in agreement with activation energies reported for overall GaAs nanowires at low temperatures using MOCVD. The decreasing of nanowire height towards higher temperature most probably the onset of competing growth on (110) side-facets and on the Au-free (111)B substrate surface. We conclude that the optimizing growth temperature is in the range of $420-$ $480^{\circ} \mathrm{C}$ with minimal tapering and reasonable uniformity of growing GaAs nanowires.

\section{Acknowledgements}

The authors acknowledge the Ministry of Science, Technology and Environment Malaysia for the financial support through Science funding 03-01-06-SF0370 and the Ibnu Sina Institute for the laboratory facilities.

\section{References}

Charles M. Lieber. (2003). Nanoscale science and technology: Building a big future from small thing, MRS Bulletin, p 486-491.

D. H. Reep, S. K. Ghandi. (1983). Journal of electrochemical society, Vol 130, p 675.

H. H. Tan, K. Sears, S. Mokkapati, Lan Fu, Yong Kim, P. McGowan, M Buda and C. Jagadish. (2006). QD and Nanowires grown by MOCVD for optoelectronic device applications, IEEE Journal of selected topics in quantum electronics, Vol 12, No 6, p 1242.

Hannah J.Joyce, Qiang Gao, H. Hoe Tan, Chennupati Jagadish, Yong Kim, Xin Zhang, Yanan Guo and Jin Zou. (2007). Twin-Free Uniform Epitaxial GaAs Nanowires Grown by a two-temperature process, Nanoletters, Vol 7, No 4, p 921-926.

James F. Shackelford. (1996). Introduction to Materials Science for Engineers, Fourth edition, Prentice Hall International Editions, p 109.

K. Hiruma, M. Yazawa, T. Katsuyama, K. Ogawa, K. Haraguchi, M. Koguchi and H. Kakibayashi. (1995). Growth and optical properties of nanometer-scale GaAs and InAs whiskers, Journal of Applied Physics, Vol 77 (2), p 447.

L. J. Lauhon, Mark S. Gudiksen and Charles M. Lieber. (2004). Semiconductor nanowire heterostructure, Phil. Trans. R. Soc. Lond, A, 362, p 1247-1260.

L. J. Lauhon, Mark S. Gudiksen and Charles M. Lieber. (2004). Semiconductor nanowire heterostructure, Phil. Trans. R. Soc. Lond, A, 362, p 1247-1260.

Lars Samuelson. (2003). Self-forming nanoscale devices, Materials today, p22.

M. Borgstrom, K. Depert, L. Samuelson, W. Seifert. (2004). Size- and shape controlled GaAs nanowhiskers grown by MOVPE : a growth study, Journal of crystal growth, Vol 260, p $18-22$.

M. C. Plante and R. R. LaPierre. (2008). Au-assisted growth of GaAs nanowires by gas source molecular beam epitaxy : Tapering, sidewall faceting and crystal structure, Journal of Crystal Growth, Vol 310, p 356-363.

M. T. Bjork et. al. (2002). Nanowire resonant tunneling diodes, Applied Physics Letter, Vol 81, Issue 23 , p 4458.

R. S. Wagner and W. C. Ellis. (1964). Vapor-liquid solid mechanism of single crystal growth, Applied Physics Letter, Vol 4, No 5, p 89.

Yue Wu, Yi Chui, Lynn Huynh, Carl J. Barrelet, David C. Bell and Charles M. Lieber. (2004). Controlled growth and structures of molecular-scale silicon nanowires, Nanoletters, Vol 4, No 3, p 433-436. 
Zhong Lin Wang, Yi Liu and Ze Zhang. (2002). Handbook of nanophase and nanostructured materials-materials system and applications (I) Vol 3, Kluwer Academic Publisher.
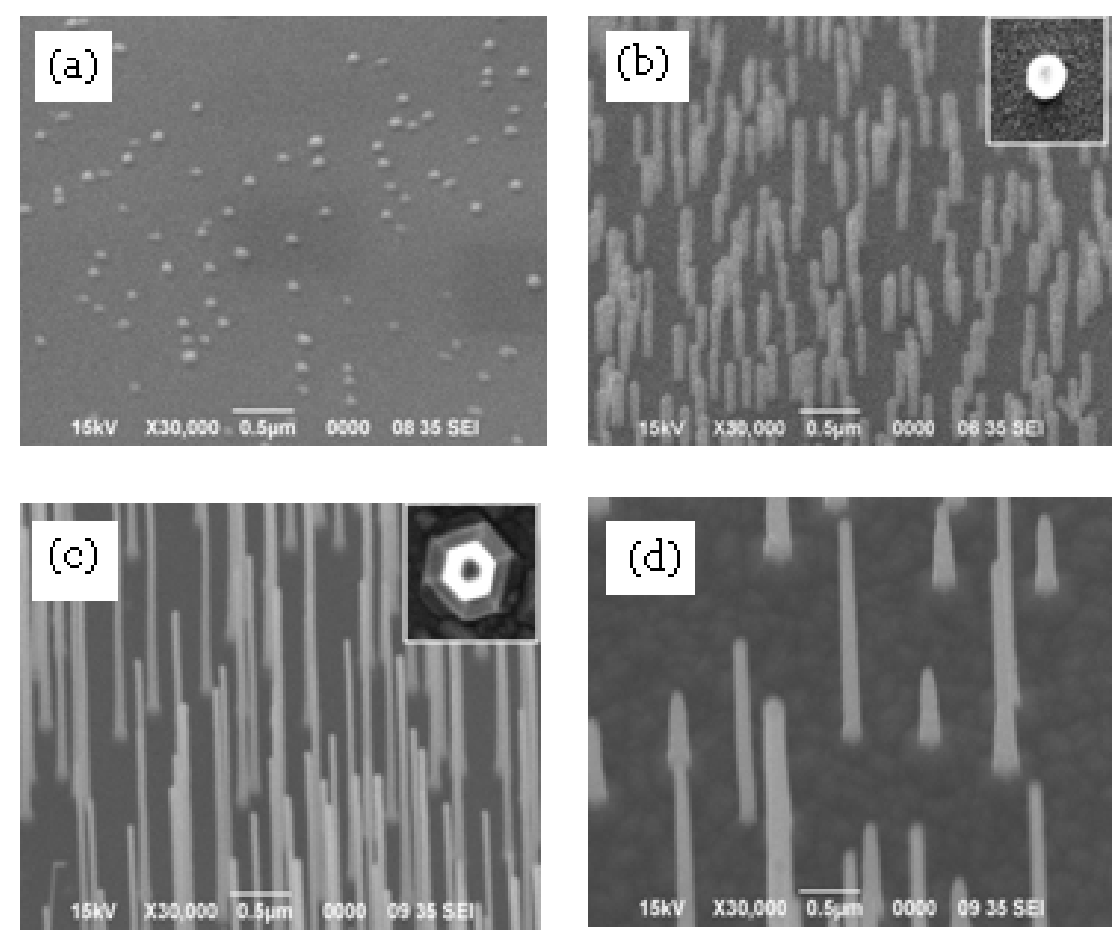

Figure 1. SEM images of GaAs nanowires grown at different temperatures: (a) $380^{\circ} \mathrm{C}$, (b) $440^{\circ} \mathrm{C}$, (c) $540^{\circ} \mathrm{C}$ and (d) $600^{\circ} \mathrm{C}$. The substrate is (111)B GaAs, tilted by $30^{\circ}$ towards the electron beam. The inset figure shows topview of a nanowire

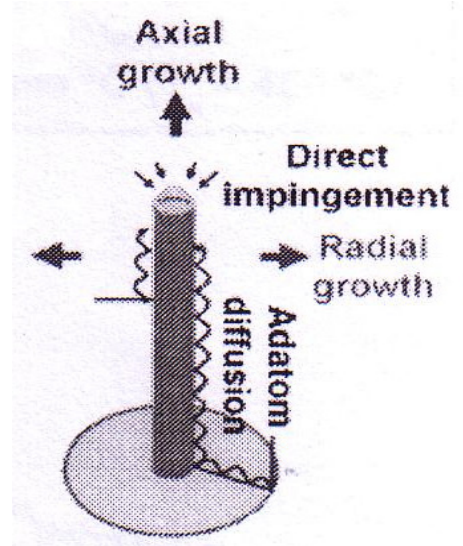

Figure 2. Schematic illustrating axial and radial nanowire growth, fed by direct impingement of precursor species on the nanoparticle and Ga adatom diffusion from the substrate and along nanowire sidewalls (Hannah J.Joyce, Qiang Gao, H. Hoe Tan, Chennupati Jagadish, Yong Kim, Xin Zhang, Yanan Guo and Jin Zou, 2007) 


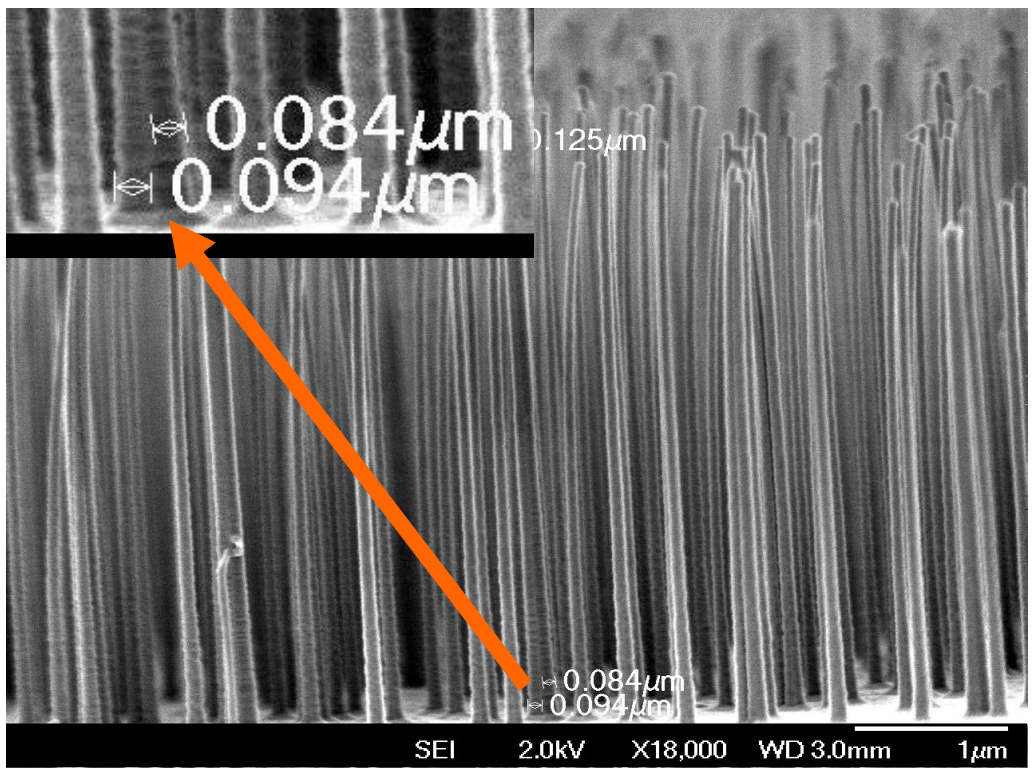

Figure 3. FE-SEM images of cross sectional GaAs nanowires grown at high temperature $540^{\circ} \mathrm{C}$ with rod-like structure at the tip and hexagonal facet at the base

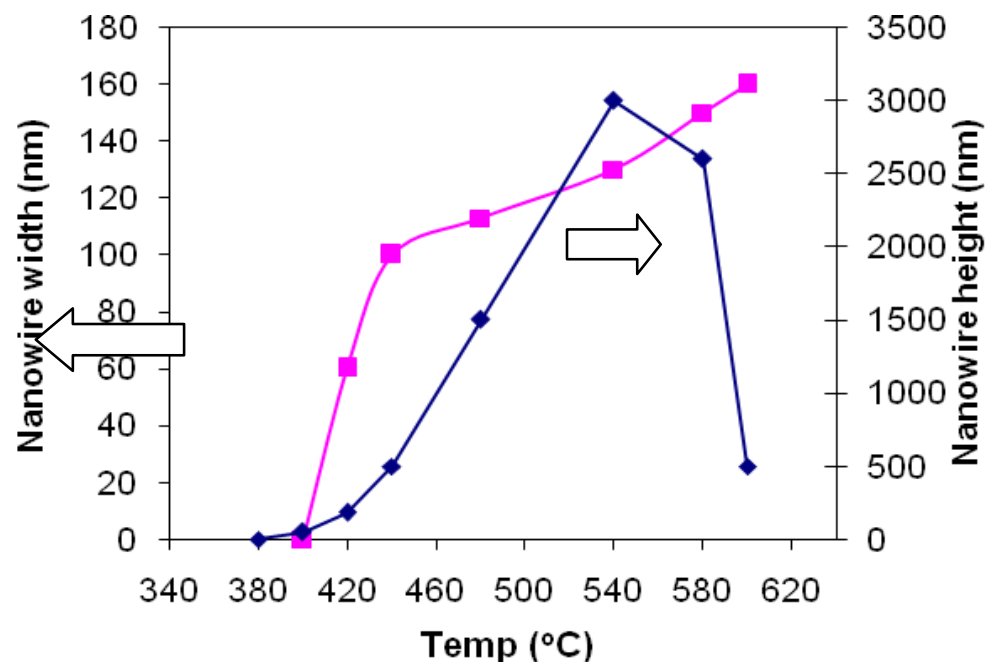

Figure 4. Width and height of GaAs nanowire as a function of the growth temperature The nanowire growth time and V/III ratio was set at 30 min and 166 respectively. 